\title{
A COMMON FIXED POINT THEOREM FOR SIX MAPPINGS WITH A NEW BINARY OPERATOR
}

\author{
Rekha Jain $^{1}$ and Saurabh jain ${ }^{2}$
}

Abstract: Using notion of compatibility, weak compatibility, commutativity and a new binary operation we have proved a fixed point theorem for six mappings satisfying a rational inequality.

2000 AMS Subject Classification: Primary: 54H25, Secondary: 47H10

Keywords: Fixed Point; Weakly compatible mappings;complete metric space; Binary operator

\section{INTRODUCTION AND PRELIMINARIES}

The concept of common fixed point theorem for commuting mapping was given by Jungck [4]. The notion of weak commutativity was introduced by Sessa [7]. Imdad and Khan [5] has proved a common fixed point theorem for six mappings which was extension of Fisher [1] [2] and Jeong- Rhoades [3]. A new binary operation was introduced by Sedghi and Shobe [6].

Definition 1.1[7]: A pair of self-mapping $(A, B)$ on a metric space $(X, d)$ is said to be weakly commuting if $d(A B x$, $B A x) \leq d(B x, A x)$ for all $x$ in $\mathrm{X}$. Obviously, commuting mappings are weakly commuting but the converse is not necessarily true.

Definition 1.2[5]: A pair of self mappings $(A, B)$ of a metric space $(X, d)$ is said to be compatible if $\lim _{n \rightarrow \infty} A x_{n}=$ $\lim _{n \rightarrow \infty} B x_{n}=t \in X$. Obviously, weakly commuting mappings are compatible but the converse is not necessarily true.

In what follows, $\mathbf{N}$ is the set of all natural numbers and $\mathbf{R}^{+}$is the set of all positive real numbers.

Let $\diamond: \mathrm{R}^{+} \times \mathrm{R}^{+} \longrightarrow \mathrm{R}^{+}$be a binary operator satisfying the following conditions:

(1) $\diamond$ is associative and commutative ;

(2) $\diamond$ is continuous ;

Some typical examples of $\diamond$ are:

\section{Examples:}

$$
\begin{gathered}
a \diamond b=\max \{a, b\} ; a \diamond b=a+b ; a \diamond b=a b+a+b ; a \diamond b=a / b(b \neq 0) ; \\
\quad \text { and } a \diamond b=\frac{a b}{\max \{a, b, 1\}} \text { for each } a, b, \in R^{+}
\end{gathered}
$$

Definition 1.3. [6]: The binary operation is said to satisfy $\alpha$-property if there exists a positive real number $\alpha$ such that

\footnotetext{
${ }^{1}$ Medi-Caps University, Indore

${ }^{2}$ Indore Institute of Science and technology
} 


$$
a \diamond b \leq \alpha \max \{a, b\}
$$

Motivated by Sedghi and Shobe [6]. and Imdad and khan[5], in the present paper, using a new binary operator we prove a fixed point theorem by improving the contraction condition and choosing suitable weak commutativity conditions .

\section{RESULTS}

We prove the following.

Theorem 2.1. Let A, B, S, T, I and J be self mappings of a complete metric space (X,d)

$$
\begin{array}{r}
\text { Satisfying } \mathrm{AB}(\mathrm{X}) \subset \mathrm{J}(\mathrm{X}), \mathrm{ST}(\mathrm{X}) \subset \mathrm{I}(\mathrm{X}) \text { and for each } x, \mathrm{y} \in \mathrm{X}, \\
d(A B x, S T y) \leq k_{1}\left[\frac{[d(A B x, J y)]^{3} \diamond[d(S T y, I x)]^{3}}{\left.[d(A B x, J y)]^{2} \diamond[d(S T y, I x)]^{2}\right]}+\right. \\
k_{2}\left[\frac{[d(A B x, J y)]^{2} \diamond[d(S T y, I x)]^{2}}{[d(A B x, J y)] \diamond[d(S T y, I x)]}\right] \\
+k_{3}[d(A B x, J y)] \diamond[d(S T y, I x)]
\end{array}
$$

if $d(A B x, J y)^{2} \diamond d(S T y, I x)^{2} \neq 0, d(A B x, J y) \diamond d(S T y, I x) \neq 0, k_{i} \geq 0 \quad(\mathrm{i}=1,2, \quad 3)$ with atleast one $k_{i}$ non zero and $2 k_{1}+2 k_{2}+2 k_{3} \alpha<1$ and

(i) $\{\mathrm{AB}, \mathrm{I}\}$ are compatible, $\mathrm{I}$ or $\mathrm{AB}$ is continuous and (ST, J) are weakly compatible or

(ii) $\{\mathrm{ST}, \mathrm{J}\}$ are compatible, $\mathrm{J}$ or $\mathrm{ST}$ is continuous and $(\mathrm{AB}, \mathrm{I})$ are weakly compatible then $\mathrm{AB}, \mathrm{ST}, \mathrm{I}$ and $\mathrm{J}$ have a unique common fixed point. Furthermore if the pairs (A, B), (A, I), (B, I), (S, T), (S, J) and (T, J) are commuting mappings then $\mathrm{A}, \mathrm{B}, \mathrm{S}, \mathrm{T}, \mathrm{I}$ and $\mathrm{J}$ have a unique common fixed point.

Proof. Let $x_{0}$ be an arbitrary point in $\mathrm{X}$. Since $\mathrm{AB}(\mathrm{X}) \subset \mathrm{J}(\mathrm{X})$ we can find a point $x_{1}$ in $\mathrm{X}$ such that $\mathrm{AB} x_{0}=\mathrm{J} x_{1}$. Also since $\mathrm{ST}(\mathrm{X})$ subset of $\mathrm{I}(\mathrm{X})$ we can choose a point $x_{2}$ with $\mathrm{ST} x_{1}=\mathrm{I} x_{2}$. Using this argument repeatedly one can construct a sequence $\left\{\mathrm{z}_{\mathrm{n}}\right\}$ such that $\mathrm{z}_{2 \mathrm{n}}=\mathrm{AB} x_{2 \mathrm{n}}=\mathrm{J} x_{2 \mathrm{n}+1}, \mathrm{z}_{2 \mathrm{n}+1}=\mathrm{ST} x_{2 \mathrm{n}+1}=\mathrm{I} x_{2 \mathrm{n}+2}$ for $\mathrm{n}=0,1,2 \ldots$ For brevity let us put

$$
\mathrm{u}_{2 \mathrm{n}}=\mathrm{d}\left(\mathrm{AB} x_{2 \mathrm{n}}, \mathrm{ST} x_{2 \mathrm{n}+1}\right) \text { and } \mathrm{u}_{2 \mathrm{n}+1}=\mathrm{d}\left(\mathrm{ST} x_{2 \mathrm{n}+1}, \mathrm{AB} x_{2 \mathrm{n}+2}\right) \text { for } \mathrm{n}=0,1,2 \ldots \ldots \text { Now we distinguish two cases }
$$

(i) Suppose that $\mathrm{u}_{2 \mathrm{n}}+\mathrm{u}_{2 \mathrm{n}+1} \neq 0$ for $\mathrm{n}=0,1,2 \ldots$

Then using the inequality (2.1), we have

$$
\begin{aligned}
& \mathrm{u}_{2 \mathrm{n}+1}=d\left(z_{2 n+1}, z_{2 n+2}\right)=d\left(S T x_{2 n+1}, A B x_{2 n+2}\right) \\
& \leq k_{1}\left[\frac{\left[d\left(A B x_{2 n+2}, J x_{2 n+1}\right)\right]^{3} \diamond\left[d\left(S T x_{2 n+1}, I x_{2 n+1}\right)\right]^{3}}{\left[d\left(A B x_{2 n+2}, J x_{2 n+1}\right)\right]^{2} \diamond\left[d\left(S T x_{2 n+1}, I x_{2 n+2}\right)\right]^{2}}\right]+ \\
& k_{2}\left[\frac{\left[d\left(A B x_{2 n+2}, J x_{2 n+1}\right)\right]^{2} \diamond\left[d\left(S T x_{2 n+1}, I x_{2 n+1}\right)\right]^{2}}{\left[d\left(A B x_{2 n+2}, J x_{2 n+1}\right)\right] \diamond\left[d\left(S T x_{2 n+1}, I x_{2 n+2}\right)\right]}\right]+ \\
& k_{3}\left[\left[d\left(A B x_{2 n+2}, J x_{2 n+1}\right)\right] \diamond\left[d\left(S T x_{2 n+1}, I x_{2 n+2}\right)\right]\right] \\
& \quad \text { or } d\left(z_{2 n+1}, z_{2 n+2}\right) \leq \frac{k_{1}+k_{2}+k_{3} \alpha}{1-\left(k_{1}+k_{2}+k_{3} \alpha\right)} d\left(z_{2 n}, z_{2 n+1}\right)
\end{aligned}
$$


Similarly we can show that

$d\left(z_{2 n}, z_{2 n+1}\right) \leq \frac{k_{1}+k_{2}+k_{3} \alpha}{1-\left(k_{1}+k_{2}+k_{3} \alpha\right)} d\left(z_{2 n-1}, z_{2 n}\right)$

thus for every $n$ we have $d\left(z_{n}, z_{n+1}\right) \leq k d\left(z_{n-1}, z_{n}\right)$

which shows that $\left\{z_{n}\right\}$ is a Cauchy sequence in the complete metric space $(\mathrm{X}, \mathrm{d})$ and so has a limit point $z$ in $\mathrm{X}$. hence the sequence $\mathrm{AB} x_{2 \mathrm{n}}=\mathrm{J} x_{2 \mathrm{n}+1}$ and $\mathrm{ST} x_{2 \mathrm{n}+1}=\mathrm{I} x_{2 \mathrm{n}+2}$ which are subsequences also converge to the point $z$.

Let us now assume that $I$ is continuous so that the sequence $\left\{I^{2} x_{2 n}\right\}$ and $\left\{I A B x_{2 n}\right\}$ converges to $I z$. Also in view of compatibility of $\{I, A B\},\left\{A B I x_{2 n}\right\}$ converges to $I z$.

Now,

$$
\begin{aligned}
d\left(A B I x_{2 n}, S T x_{2 n+1}\right) \leq & k_{1}\left[\frac{\left[d\left(A B I x_{2 n}, J x_{2 n+1}\right)\right]^{3} \diamond\left[d\left(S T x_{2 n+1}, I^{2} x_{2 n}\right)\right]^{3}}{\left[d\left(A B I x_{2 n}, J x_{2 n+1}\right)\right]^{2} \diamond\left[d\left(S T x_{2 n+1}, I^{2} x_{2 n}\right)\right]^{2}}+\right. \\
& k_{2}\left[\frac{\left.\left[d\left(A B I x_{2 n}, J x_{2 n+1}\right)\right]^{2} \diamond\left[d\left(S T x_{2 n+1}, I^{2} x_{2 n}\right)\right]^{2}\right]}{\left[d\left(A B I x_{2 n}, J x_{2 n+1}\right)\right] \diamond\left[d\left(S T x_{2 n+1}, I^{2} x_{2 n}\right)\right]}+\right. \\
& k_{3}\left[d\left(A B I x_{2 n}, J x_{2 n+1}\right)\right] \diamond\left[d\left(\operatorname{STx}_{2 n+1}, I^{2} x_{2 n}\right)\right]
\end{aligned}
$$

Which on letting $n \rightarrow \infty$ reduces to

$$
\left(1-k_{1}-k_{2}-k_{3} \alpha\right) d(I z, z) \leq 0
$$

Now,

$$
\text { yielding thereby } I z=z
$$

$$
\begin{aligned}
d\left(A B z, S T x_{2 n+1}\right) \leq & k_{1}\left[\frac{\left[d\left(A B z, J x_{2 n+1}\right)\right]^{3} \diamond\left[d\left(S T x_{2 n+1}, I z\right)\right]^{3}}{\left[d\left(A B z, J x_{2 n+1}\right)\right]^{2} \diamond\left[d\left(S T x_{2 n+1}, I z\right)\right]^{2}}\right]+ \\
& k_{2}\left[\frac{\left[d\left(A B z, J x_{2 n+1}\right)\right]^{2} \diamond\left[d\left(S T x_{2 n+1}, I z\right)\right]^{2}}{\left[d\left(A B z, J x_{2 n+1}\right)\right] \diamond\left[d\left(S_{2 n+1}, I z\right)\right]}\right]+ \\
& k_{3}\left[d\left(A B z, J x_{2 n+1}\right)\right] \diamond\left[d\left(\operatorname{STx}_{2 n+1}, I z\right)\right]
\end{aligned}
$$

On letting $n \rightarrow \infty$ and using $I z=z \quad$ we get

$$
d(A B z, z) \leq\left(k_{1}+k_{2}+k_{3} \alpha\right) d(A B z, z)
$$

This implies $A B z=z$

Since $\quad A B(X) \subset J(X)$ then there always exists a point $z^{\prime}$ such that $J z^{\prime}=z$ so that

$S T z=S T\left(J z^{\prime}\right)$.

Now,

$$
d(Z, S T z)=d(A B z, S T z)
$$




$$
\begin{aligned}
\leq & k_{1}\left[\frac{\left[d\left(A B z, J z^{\prime}\right)\right]^{3} \diamond\left[d\left(S T z^{\prime}, I z\right)\right]^{3}}{\left[d\left(A B z, J z^{\prime}\right)\right]^{2} \diamond\left[d\left(S T z^{\prime}, I z\right)\right]^{2}}\right]+ \\
& k_{2}\left[\frac{\left[d\left(A B z, J z^{\prime}\right)\right]^{2} \diamond\left[d\left(S T z^{\prime}, I z\right)\right]^{2}}{\left[d\left(A B z, J z^{\prime}\right)\right] \diamond\left[d\left(S T z^{\prime}, I z\right)\right]}\right]+ \\
& k_{3}\left[d\left(A B z, J z^{\prime}\right)\right] \diamond\left[d\left(S T z^{\prime}, I z\right)\right] \\
\leq & \left(k_{1}+k_{2}+k_{3} \alpha\right)\left[d\left(S T z^{\prime}, z\right)\right]
\end{aligned}
$$

Hence, $S T z^{\prime}=z=J z^{\prime}$ which shows that $z^{\prime}$ is the coincidence point of $S T$ and $J$. Now using the weak compatibility of $(S T, J)$, now using the weak compatibility of $(S T, \quad J)$, we have

$$
S T z=S T\left(J z^{\prime}\right)=J\left(S T z^{\prime}\right)=J z
$$

which shows that $z$ is also a coincidence point of the pair (ST, J). Now

$$
\begin{gathered}
d(z, S T z)=d(A B z, S T z) \\
\leq k_{1}\left[\frac{[d(A B z, J z)]^{3} \diamond[d(S T z, I z)]^{3}}{[d(A B z, J z)]^{2} \diamond[d(S T z, I z)]^{2}}\right]+ \\
k_{2}\left[\frac{[d(A B z, J z)]^{2} \diamond[d(S T z, I z)]^{2}}{[d(A B z, J z)] \diamond[d(S T z, I z)]}\right]+ \\
k_{3}[d(A B z, J z)] \diamond[d(S T z, I z)] \\
\leq\left(k_{1}+k_{2}+k_{3} \alpha\right) d(z, S T z)
\end{gathered}
$$

Hence, $z=S T z=J z$ which shows that $z$ is a common fined point of $A B, I, S T$ and $J$.

Now suppose that $A B$ is continuous so that the sequences $\left\{A B^{2} x_{2 n}\right\}$ and $\left\{A B I x_{2 n}\right\}$ converge to $A B z$ since $(A B, I)$ are compatible it follows that $\left\{I A B x_{2 n}\right\}$ also converge to $A B z$, thus

$$
\begin{aligned}
d\left(A B^{2} x_{2 n}, S T x_{2 n+1}\right) \leq & k_{1}\left[\frac{\left[d\left(A B^{2} x_{2 n}, J x_{2 n+1}\right)\right]^{3} \diamond\left[d\left(S T x_{2 n+1}, I A B x_{2 n}\right)\right]^{3}}{\left[d\left(A B^{2} x_{2 n}, J x_{2 n+1}\right)\right]^{2} \diamond\left[d\left(S T x_{2 n+1}, I A B x_{2 n}\right)\right]^{2}}+\right. \\
& k_{2}\left[\frac{\left[d\left(A B^{2} x_{2 n}, J x_{2 n+1}\right)\right]^{2} \diamond\left[d\left(S T x_{2 n+1}, I A B x_{2 n}\right)\right]^{2}}{\left[d\left(A B^{2} x_{2 n}, J x_{2 n+1}\right)\right] \diamond\left[d\left(S T x_{2 n+1}, I A B x_{2 n}\right)\right]}\right]+ \\
& k_{3}\left[d\left(A B^{2} x_{2 n}, J x_{2 n+1}\right)\right] \diamond\left[d\left(S T x_{2 n+1}, I A B x_{2 n}\right)\right]
\end{aligned}
$$

which on letting $n \rightarrow \infty$ reduces to

$$
d(A B z, z) \leq\left(k_{1}+k_{2}+k_{3} \alpha\right) d(A B z, z)
$$

which implies $A B z=z$

as earlier, there exists $z^{\prime}$ is $X$ such that $A B z=z=J z^{\prime}$ then 


$$
\begin{gathered}
d\left(A B^{2} x_{2 n}, S T z^{\prime}\right) \leq k_{1}\left[\frac{\left[d\left(A B^{2} x_{2 n}, J z^{\prime}\right)\right]^{3} \diamond\left[d\left(S T z^{\prime}, I A B x_{2 n}\right)\right]^{3}}{\left[d\left(A B^{2} x_{2 n}, J z^{\prime}\right)\right]^{2} \diamond\left[d\left(S T z^{\prime}, I A B x_{2 n}\right)\right]^{2}}\right]+ \\
k_{2}\left[\frac{\left.\left[d\left(A B^{2} x_{2 n}, J z^{\prime}\right)\right]^{2} \diamond\left[d\left(S T z^{\prime}, I A B x_{2 n}\right)\right]^{2}\right]}{\left[d\left(A B^{2} x_{2 n}, J z^{\prime}\right)\right] \diamond\left[d\left(S T z^{\prime}, I A B x_{2 n}\right)\right]}\right] \\
k_{3}\left[d\left(A B^{2} x_{2 n}, J z^{\prime}\right)\right] \diamond\left[d\left(S T z^{\prime}, I A B x_{2 n}\right)\right] \\
\quad \begin{array}{c}
\text { which on letting } n \rightarrow \infty \text { reduces to } \\
d\left(z, S T z^{\prime}\right) \leq\left(k_{1}+k_{2}+k_{3} \alpha\right) d\left(z, S T z^{\prime}\right)
\end{array}
\end{gathered}
$$

This gives $S T z^{\prime}=z=J z^{\prime}$ thus $z^{\prime}$ is a coincidence point of $(S T, J)$. Since, the pair $(S T, J)$ is weakly compatible hence $S T z=S T\left(J z^{\prime}\right)=J\left(S T z^{\prime}\right)=J z$ which shows that $S T z=J z$. Further

$$
\begin{aligned}
d\left(A B x_{2 n}, S T z\right) \leq k_{1} & {\left[\frac{\left[d\left(A B x_{2 n}, J z\right)\right]^{3} \diamond\left[d\left(S T z, I x_{2 n}\right)\right]^{3}}{\left.\left[d\left(A B x_{2 n}, J z\right)\right]^{2} \diamond\left[d\left(S T z, I x_{2 n}\right)\right]^{2}\right]+}\right.} \\
& k_{2}\left[\frac{\left[d\left(A B x_{2 n}, J z\right)\right]^{2} \diamond\left[d\left(S T z, I x_{2 n}\right)\right]^{2}}{\left[d\left(A B x_{2 n}, J z\right)\right] \diamond\left[d\left(S T z, I x_{2 n}\right)\right]}\right]+ \\
& k_{3}\left[d\left(A B x_{2 n}, J z\right)\right] \diamond\left[d\left(S T z, I x_{2 n}\right)\right]
\end{aligned}
$$

which on letting $n \rightarrow \infty$ reduces to

$$
d(z, S T z) \leq\left(k_{1}+k_{2}+k_{3} \alpha\right) d(z, S T z)
$$

It follows that $S T z=z=J z$

The point $z$ therefore is in the range of $S T$ and since $S T(X) \subset I(X)$ there exist a point $z$ " in $X$ such that

$I z^{\prime \prime}=z$. Thus

$$
\begin{aligned}
d\left(A B z^{\prime \prime}, z\right)=d\left(A B z^{\prime \prime}, S T z\right) \leq & k_{1}\left[\frac{\left[d\left(A B z^{\prime \prime}, J z\right)\right]^{3} \diamond\left[d\left(S T z, I z^{\prime \prime}\right)\right]^{3}}{\left[d\left(A B z^{\prime \prime}, J z\right)\right]^{2} \diamond\left[d\left(S T z, I z^{\prime \prime}\right)\right]^{2}}\right]+ \\
& k_{2}\left[\frac{\left.\left[d\left(A B z^{\prime \prime}, J z\right)\right]^{2} \diamond\left[d\left(S T z, I z^{\prime \prime}\right)\right]^{2}\right]}{\left[d\left(A B z^{\prime \prime}, J z\right)\right] \diamond\left[d\left(S T z, I z^{\prime \prime}\right)\right]}\right]+ \\
& k_{3}\left[d\left(A B z^{\prime \prime}, J z\right)\right] \diamond\left[d\left(S T z, I z^{\prime \prime}\right)\right]
\end{aligned}
$$

Letting $n \rightarrow \infty$

$$
d\left(A B z^{\prime \prime} z\right) \leq\left(k_{1}+k_{2}+k_{3} \alpha\right) d\left(A B z^{\prime \prime}, z\right)
$$

which shows that $A B z^{\prime \prime}=z$

Also since $(A B, I)$ are compatible and hence using weakly commuting we obtain

$$
\begin{array}{r}
d(A B z, I z)=d\left(A B(I z "), I\left(A B z^{\prime \prime}\right)\right) \\
\leq d(I z ", A B z ")=d(z, z)=0
\end{array}
$$


Therefore $A B z=I z=z$

Thus we have proved that $z$ is common fixed point of $A B, S T, I$ and $J$.

If the mappings $S T$ or $J$ is continuous instead of $A B$ or $I$ then proof of $z$ is a common fixed point of $A B, S T, I$ and $J$ is similar.

Let $v$ be another fixed point of $I, J, A B$ and $S T$

then

$$
d(z, v)=d(A B z, S T v)
$$

$$
\begin{gathered}
\leq k_{1}\left[\frac{\left.[d(A B z, J v)]^{3} \diamond[d(S T v, I z)]^{3}\right]}{[d(A B z, J v)]^{2} \diamond[d(S T v, I z)]^{2}}\right]+ \\
k_{2}\left[\frac{[d(A B z, J v)]^{2} \diamond[d(S T v, I z)]^{2}}{[d(A B z, J v)] \diamond[d(S T v, I z)]}\right]+ \\
k_{3}[d(A B z, J v)] \diamond[d(S T v, I z)] \\
d(z, v) \leq\left(k_{1}+k_{2}+k_{3} \alpha\right) d(z, v) \text { yielding thereby } z=v
\end{gathered}
$$

Finally we need to show that $z$ is also a common fixed point of $A, B, S, T, I$ and $J$. For this let $z$ be the unique common fixed point of both the pairs $(A B, I)$ and $(S T, J)$. Then

$$
\begin{aligned}
& A z=A(A B z)=A(B A z)=A B(A z), A z=A(l z)=I(A z) \\
& B z=B(A B z)=B(A(B z))=B A(B z)=A B(B z) . \quad B z=B(l z)=I(B z)
\end{aligned}
$$

which shows that $A z$ and $B z$ is a common fixed point of $(A B, I)$ yielding thereby $A z=z=B z=l z=A B z$ in the view of uniqueness of the common fixed point of the pair $(A B, l)$.

Similarly using the commutatively of $(S, T),(S J)$ and $(T, J)$ it can be shown that $S z=z=T z=J z=S T z$.

Now we need to show that $A z=S z(B z=T z)$ also remains a common fixed point of both the pairs $(A B, I)$ and $(S T, J)$. For this

$$
\begin{aligned}
& d(A z, S z)=d(A(B A z), S(T S z)) \\
& =d(A B(A z), S T(S z))
\end{aligned}
$$




$$
\begin{gathered}
\leq k_{1}\left[\frac{[d(A B(A z), J(S z))]^{3} \diamond[d(S T(S z), I(A z))]^{3}}{\left.[d(A B(A z), J(S z))]^{2} \diamond[d(S T(S z), I(A z))]^{2}\right]+}\right. \\
k_{2}\left[\frac{[d(A B(A z), J(S z))]^{2} \diamond[d(S T(S z), I(A z))]^{2}}{[d(A B(A z), J(S z))] \diamond[d(S T(S z), I(A z))]}\right]+ \\
k_{3}[d(A B(A z), J(S z))] \diamond[d(S T(S z), I(A z))]
\end{gathered}
$$

Which implies that $d(A z, S z)=0 \quad($ as $d(A B(A z), J(S z)+d(S T(S z), I(A z))=0)$ yielding thereby $A z=S z$. Similarly it can be shown that $B z=T z$. Thus $z$ is the unique common fixed of $A, B, S, T, l$ and $J$.

Corollary 2.1. Theorem 2.1 remains true if contraction conditions (2.1) and (2.2) any of the following conditions:

$$
\begin{aligned}
& \text { (i) } d(A B z, S T y) \leq k_{1}\left[\frac{[d(A B x, J y)]^{3} \diamond[d(S T y, I x)]^{3}}{[d(A B x, J y)]^{2} \diamond[d(S T y, I x)]^{2}}\right] \\
& +k_{3}[d(A B x, J y)] \diamond[d(S T y, I x)] \\
& \text { if }[d(A B x, J y)]^{2} \diamond[d(S T y, l x)]^{2} \neq 0, \quad k_{1}, k_{3}>0,2 k_{1}+2 k_{3} \alpha_{3}<1 \text { or } \\
& d(A B x, S T y)=0 \text { if }[d(A B x, J y)]^{2}+[d(S T y, l x)]^{2}=0 \\
& \text { (ii) } d(A B x, S T y) \leq k_{1}\left[\frac{[d(A B x, J y)]^{3} \diamond[d(S T y, I x)]^{3}}{[d(A B x, J y)]^{2} \diamond[d(S T y, I x)]^{2}}\right]+k_{2}\left[\frac{[d(A B x, J y)]^{2} \diamond[d(S T y, I x)]^{2}}{[d(A B x, J y)] \diamond[d(S T y, I x)]}\right] \\
& \text { if }[d(A B x, J y)]^{2} \diamond[d(S T y, I x)]^{2} \neq 0,[d(A B x, J y)] \diamond[d(S T y, I x)] \neq 0, k_{1}, k_{2}>0,2 k_{1}+2 k_{2}<1 \\
& \text { (iii) Either } d(A B x, S T y) \leq k_{1}\left[\frac{[d(A B x, J y)]^{3} \diamond[d(S T y, I x)]^{3}}{[d(A B x, J y)]^{2} \diamond[d(S T y, I x)]^{2}}\right] \\
& {\left[[d(A B x, J y)]^{2} \diamond[d(S T y, I x)]^{2}\right] \neq 0, k_{1}>0, k_{1}<1 / 2}
\end{aligned}
$$

Proof. Corollaries corresponding to the contraction conditions (A), (B) and (C) can be deduced directly from. Theorem 2.1 by choosing $k_{2}=0, k_{3}=0$ and $k_{2}=k_{3}=0$, respectively.

\section{REFERENCES}

[1]. Fisher B. Mapping satisfying rational inequality, Nanta. Math. 12 (1979) $195-199$.

[2]. Fisher B. Common fixed point and constant mapping satisfying a rational inequality, Math. Sem. Notes 6 (1978) 29 -35.

[3]. Imdad. M and Khan. Q. H, a common fixed point theorem for six mapping satisfying a rational inequality, Ind. Jou. of math. 44 (2002) 47-57. 
[4]. Jeong G. S. and B. E. Rhoades. Some remark for improving fixed point theorem for more than two maps, Indain J. Pure Appl. Math. 28 (1997) 1177 - 1196.

[5]. Jungck. G. Compatible mappings and Common fixed points, Internat. J. Math. and Math. Sci. 9 (1986) 771 779.

[6]. Sedghi S. and Shobe N. Common fixed point theorems for four mappings in complete metric spaces, Bulletin of the Iranian Math. Soc. Vol. 33 (2) (2007), 37 - 47.

[7]. Sessa $\mathrm{S}$, a weak commutativity condition of mappings in fixed point considerations, Pub. Inst. Math. (Beogard) 32 (1982) 149-153. 